Revista de Comunicación y Salud, 2022, Vol. 12, 21-44

Editado por Cátedra de Comunicación y Salud

ISSN: 2173-1675

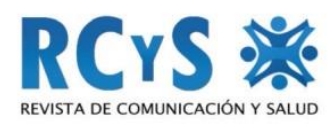

Enviado 14/04/2021

Aprobado 13/09/2021

Publicado 03/01/2022

\title{
ANÁLISIS DE LA HUMANIZACIÓN EN EL ÁMBITO SANITARIO EN REDES SOCIODIGITALES
}

\author{
Analysis of humanization in the health field in digital social networks \\ María del Mar Simón Márquez \\ Universidad de Almería. España. \\ msm112@ual.es. \\ María del Mar Molero Jurado \\ Universidad de Almería. España. \\ mmj130@ual.es. \\ Ana Belén Barragán Martín \\ Universidad de Almería. España. \\ abm410@ual.es. \\ África Martos Martínez \\ Universidad de Almería. España. \\ amm521@ual.es. \\ María del Carmen Pérez-Fuentes \\ Universidad de Almería. España. \\ mpf421@ual.es. \\ José Jesús Gázquez Linares \\ Universidad de Almería. España. \\ jlinares@ual.es
}

Financiación. Se introducirá una breve nota sobre las fuentes de financiación en el caso de que el artículo esté vinculado a un proyecto de investigación financiado por alguna institución pública (planes nacionales de $1+D+i$, convocatorias de universidades, gobiernos regionales, etc.) o privada.

\section{Cómo citar el artículo}

Simón Márquez, M. M., Molero Jurado, M.M., Barragán Martín, A. B., Martos Martínez, A., Pérez-Fuentes, M.C. y Gázquez Linares, J.J. (2022). Análisis de la humanización en el ámbito sanitario en redes sociodigitales. Revista de Comunicación y Salud, 12, 21-44. https://doi.org/10.35669/rcys.2022.12.e282 


\title{
Resumen
}

La humanización en la atención implica un conjunto de recursos sociales, personales y emocionales en el profesional sanitario que faciliten el desarrollo de su labor y les posibilite tener un papel activo en la recuperación de los pacientes. Por ello, es imprescindible el papel de las redes sociodigitales para la formación e información en humanización dentro del ámbito sanitario, donde se creen debates y manifiesten la importancia de la humanización en el entorno sanitario. Por todo ello, es analizar la presencia de la humanización en redes sociales a través de entradas de Twitter y Facebook, como reflejo de la influencia en población general, de Estados Unidos, España, Italia, Colombia, Brasil, Portugal, Canadá y Francia. En total, se analizaron 154 tuits y 23 entradas de Facebook, todos aquellos relacionados con la humanización en el ámbito sanitario. Tras el análisis de las diferentes entradas en las redes sociodigitales, se muestran diferentes fuentes que expresan opiniones sobre la humanización hospitalaria desde los propios hospitales, congresos dirigidos exclusivamente a esta temática, pacientes, familiares, fundaciones, asociaciones, sindicatos, entre otros. La mayoría de entradas tanto en Facebook como Twitter, hablan de la importancia de la humanización en cuidados y el trato dirigido tanto al paciente como a sus familiares y a los propios profesionales sanitarios, siendo imprescindibles en la humanización en cuidados dentro del sistema sanitario. Así mismo, reivindican desde todos los ámbitos sociales la poca visibilidad que se le da a la humanización en cuidados. Además, se observa la preocupación y el interés que genera la humanización las Unidades de Cuidados Intensivos. De forma general, se muestra cómo las redes sociodigitales son de vital importancia para la comunicación e intercambio de información y conocimientos sobre la humanización, una temática que comienza a suscitar interés a nivel social dentro del ámbito sanitario.

Palabras clave: humanización, salud, cuidados, redes sociodigitales, comunicación.

\begin{abstract}
The humanization of care implies a set of social, personal and emotional resources in the health professional that facilitate the development of their work and enable them to play an active role in the recovery of patients. For this reason, the role of socio-digital networks for training and information on humanization within the health field is essential, where debates are created and the importance of humanization in the health environment is manifested. Therefore, the objective of this work is to analyze the presence of humanization in social networks through Twitter and Facebook entries, as a reflection of the influence on the general population, the United States, Spain, Italy, Colombia, Brazil, Portugal, Canada and France. In total, 154 tweets and 23 Facebook entries were analyzed, all those related to humanization in the health field. After the analysis of the different entries in the socio-digital networks, different sources are shown that express opinions on hospital humanization from the hospitals themselves, congresses aimed exclusively at this issue, patients, family members, foundations, associations, unions, among others. Most of the entries on both Facebook and Twitter
\end{abstract}


speak of the importance of humanization in care and the treatment directed both to the patient and their families and to the health professionals themselves, being essential in the humanization of care within the health system. Likewise, they claim from all social spheres the little visibility that is given to humanization in care. In addition, the concern and interest generated by the humanization of the Intensive Care Units is observed. In general, it shows how socio-digital networks are of vital importance for the communication and exchange of information and knowledge about humanization, a topic that is beginning to arouse interest at the social level within the health field.

Keywords: humanization, health, care, digital social networks, communication.

\section{INTRODUCCIÓN}

La humanización en la atención implica un conjunto de recursos sociales, personales y emocionales en el profesional sanitario que faciliten el desarrollo de su labor y les posibilite tener un papel activo en la recuperación de los pacientes. Un proceso en el cual, se tenga en cuenta el bienestar y las necesidades de las personas (García-Salido et al., 2019), mostrando así, interés y empatía por las preocupaciones y temores (Calegari et al., 2015). Así mismo, los profesionales sanitarios proporcionan herramientas con la finalidad de mejorar la salud de los mismos (Luiz et al, 2017; Nora y Junges, 2013; Tripodi et al., 2019).

Así bien, la humanización queda definida como una serie de competencias personales que hacen posible desarrollar la actividad profesional dentro del ámbito de la asistencia sanitaria, cuidando, atendiendo y respetando al ser humano. Por ello, la humanización se centra en la mejora de la atención tanto física, como mental y emocional, dirigida no solo a los pacientes sino también a los profesionales sanitarios, siendo estos dos imprescindibles en la humanización en cuidados (Pérez-Fuentes et al., 2019a; Pérez-Fuentes et al., 2019b).

El objetivo de la humanización en cuidados es garantizar una excelente atención a los pacientes, satisfaciendo así las necesidades de los mismos durante su estancia hospitalaria (Borbasi et al., 2012; Pérez-Fuentes et al., 2018). Para ello, los profesionales sanitarios deben estar comprometidos con su trabajo, repercutiendo este en la mejora de resultados en cuanto a la relación al paciente se refiere (Carli et al., 2018; Pérez-Fuentes et al., 2018).

Por todo ello, la humanización en el profesional sanitario se relaciona con aspectos como la afectación, la autoeficacia, la comprensión emocional, la disposición al optimismo y la sociabilidad (Pérez-Fuentes et al., 2019b).

La humanización en cuidados en el profesional sanitario implica una actitud optimista, mediante la cual, solvente los problemas a los que se enfrenta en su vida generando expectativas positivas ya que se encuentra motivados para alcanzar los objetivos que se proponen (Di Fabio et al., 2018; Segerstrom et al., 2017). El optimismo 
en la profesión sanitaria puede propiciar una mejora de su desempeño laboral, así como de las relaciones sociales y la salud física y psicológica (Carver y Scheier, 2014; Seligman et al., 2007).

Por otro lado, es usual que el profesional sanitario posea habilidades sociables debido a la capacidad que tiene para tratar con el paciente y sus familiares incrementando así su desempeño laboral. Además, cooperar en equipo en este ámbito es fundamental para el desarrollo de un trabajo eficaz donde se lleve a cabo una atención centrada en la persona (Lown et al., 2016; Molero et al., 2018a).

Así mismo, la autoeficacia en el profesional sanitario es otro de los factores relacionados con la humanización. Esta es entendida como las expectativas que el individuo tiene de sí mismo sobre sus capacidades, de manera que dirija y ejecute acciones con la finalidad de conseguir el rendimiento estimado (Bandura et al., 1987). Así como ser capaz de manejar y expresar sus propias emociones, lo que implica enfrentarse a situaciones concretas que requieren una acción adaptada en función de la persona y el contexto mejorando así la calidad en la atención sanitaria (Molero et al., 2018a; Pérez-Fuentes et al., 2019a). Además, cabe destacar la relación existente entre la autoestima, inteligencia emocional y autoeficacia, debido a que estas se asocian con la capacidad que tiene el individuo para comunicarse y relacionarse con las personas de su entorno (Pérez-Fuentes et al., 2019c).

Los profesionales sanitarios se enfrentan a menudo a situaciones estresantes derivadas de situaciones complejas acontecidas en su lugar de trabajo. Este tipo de situaciones pueden generar en el trabajador ansiedad y sentimiento de culpa si no superan con éxito el problema al que se enfrentan. Por ello, es fundamental la autorregulación de las emociones en el que el control de la afectación, para garantizar la calidad de la atención y el bienestar del propio profesional (García-Rodríguez et al., 2015; Pérez-Fuentes et al., 2019a; Soriano et al., 2019; Wersebe et al., 2019).

Por último, se encuentra el compromiso emocional en la humanización en cuidados, referido a la capacidad que tienen las personas de entenderse a sí mismas y a otras personas, de modo que se mantenga una consonancia lógica entre lo que se piensa, lo que se siente y lo que finalmente se decide hacer, comprendiendo y gestionando de manera eficaz las emociones como personas emocionalmente inteligentes (Bartosiewicz y Januszewicz, 2019; Howick et al., 2017; Pérez-Fuentes et al., 2019d).

\subsection{Influencia de la humanización en las redes sociodigitales}

De la necesidad de formación e información en humanización dentro del ámbito sanitario, en las redes sociodigitales se comienza a hacer uso de este concepto, donde se crean debates y, se manifiesta la importancia de la humanización en el entorno sanitario. Es por ello, que las redes sociodigitales tienen un papel crucial en el desarrollo de la humanización en cuidados dentro del ámbito sanitario. A raíz de esta carencia informativa en fuentes de calidad científica, las redes sociodigitales se han convertido en una opción para acercarse a la sanidad (Penni, 2017).

Revista de Comunicación y Salud, 2022, Vol. 12, 21-44 
El uso de internet ha generado nuevas alternativas para las redes sociodigitales, que han ido cambiando y se han ajustado para lograr satisfacer las necesidades y demandas de la población (Erfani y Abedin, 2018). Las redes sociodigitales establecen conexiones entre personas, instituciones, organizaciones, entidades políticas, sanitarios, y una multitud de unidades. Por ello, se originan interacciones comunicativas entre las personas que utilizan estos medios para expresar opiniones o compartir ideas, recursos materiales, información, conocimiento sobre diferentes temas de interés (Anwar et al., 2019).

Las características más reseñables de las redes sociodigitales son el fácil acceso (Bendayan y Blanca, 2019; Klimova y Pikhart, 2020), la creación de vínculos humanos, la interactividad, la comunicación inmediata y continua, no siendo necesaria la experiencia para saber utilizarlas. Las redes sociodigitales han facilitado e incrementado la comunicación entre personas a nivel mundial (Mehdipour, 2019).

En el ámbito sanitario, las redes sociodigitales resultan útiles para conocer los servicios que ofrecen los diferentes centros sanitarios, no obstante, cabe destacar como en múltiples ocasiones las personas hacen uso de sus redes sociodigitales para consultar sus dolencias antes de ponerse en contacto con el sistema de salud (Amoah et al., 2018).

En las redes sociodigitales, se crean vínculos a través de actividades compartidas, ideas, intercambio de opiniones, debates, compartir sentimientos, entre otros. Este tipo de vínculos llegan a crear diferentes tipos de apoyo como por ejemplo el emocional, instrumentar o informativo (Perkins et al., 2015).

En el campo de la salud, las redes sociodigitales posibilitan la comunicación con otras personas que tienen las mismas incertidumbres sobre los problemas de salud que se les plantean o manifiestan los déficits que presentan los sistemas sanitarios en ciertos ámbitos. Esto puede llegar a originar cambios en el mismo, generando así protestas masivas que influyan en la política de salud para provocar, cambios que mejoren el sistema sanitario (Griffiths et al., 2015).

La comunicación a través de las redes sociodigitales, ha generado un cambio en la manera en la que las personas acceden y obtienen información sobre temas del entorno sanitario, se ha convertido en un espacio donde se intercambian datos de salud desde la propia experiencia personal y dónde consiguen una respuesta inmediata que les brinda tranquilidad y apoyo (Ziebland y Wyke, 2012; Griffiths et al., 2012). Las redes sociodigitales como Facebook o Twitter son un servicio gratuito y rápido que permite a los usuarios a través de publicaciones, difundir información sobre las carencias de los sistemas sanitarios, suponiendo una nueva perspectiva, sobre todo en la actualidad donde los medios de comunicación no cubren ciertas informaciones sanitarias (García Del Castillo et al., 2020). 
No obstante, se debe tener en cuenta que la información que se muestra en redes sociodigitales en su mayoría no ha sido contrastada, por lo que puede contener información errónea sobre la salud que puede divulgarse fácilmente (Scanfeld et al., 2010).

A través de las redes, se crean diversos grupos de red digital entre los que se encuentran familias, personas con enfermedades raras, o con enfermedades crónicas, entre otros, que aprovechan este recurso para conseguir un sistema sanitario eficiente y de calidad, proporcionando así un planteamiento innovador con la finalidad de mejorar las políticas de los servicios de salud (Griffiths et al., 2015).

\section{OBJETIVO}

El objetivo del presente trabajo es analizar la presencia de la humanización en redes sociales a través de entradas de Twitter y Facebook, como reflejo de la influencia en población general, de Estados Unidos, España, Italia, Colombia, Brasil, Portugal, Canadá y Francia.

\section{METODOLOGÍA}

\subsection{Diseño}

El presente trabajo se basa en un procedimiento sistemático de revisión y análisis de corte cualitativo, con el objetivo de identificar la información existente en redes sobre la humanización en el ámbito sanitario. Se seleccionó la metodología cualitativa porque permite analizar fragmentos de una manera global y contextualizada (Pizarro, 2000). El estudio trata de analizar a través de publicaciones sobre humanización en salud en redes sociodigitales, concretamente Twitter y Facebook.

\subsection{Producción de datos}

La recopilación de publicaciones se realizó entre el 8 de diciembre de 2019 y el 21 de diciembre de 2020. Así bien, se recopilaron todas aquellas publicaciones que contuviesen los descriptores "humanización", "humanización en cuidados", "humanización en gestión", "humanización en pediatría", "humanización en farmacia", limitando el idioma español, portugués, francés, inglés, e italiano. Obteniendo posteos de diferentes países como Estados Unidos, España, Italia, Colombia, Brasil, Portugal, Canadá y Francia.

Como criterios de exclusión de las publicaciones fueron: Retuits, publicaciones compartidas, menciones, publicaciones no relacionadas con la humanización en cuidados, y cuentas de acceso privado. Por otro lado, como criterios de inclusión, se escogen los Tweet y las entradas de Facebook publicadas que informan sobre la humanización en cuidados, así como las publicaciones que provengan de perfiles públicos y de acceso abierto. 
Tras aplicar los diferentes criterios de exclusión e inclusión se analizaron un total de 154 tweet y 23 entradas de Facebook.

En la Tabla 1, se muestran las publicaciones seleccionadas en función de la categoría procedente entre los que destacamos Asociaciones $(n=9)$, Hospitales $(n=30)$, Usuarios $(n=95)$, Portales Sanitarios $(n=14)$, Fundaciones $(n=8)$, Empresas sanitarias $(n=3)$, instituciones $(n=7)$, Medios de información $(n=6)$, Congresos $(n=5)$.

Tabla 1. Recopilación de publicaciones en función del medio obtenido

\begin{tabular}{|l|c|}
\hline Categorías & Número de publicaciones \\
\hline Asociaciones & 9 \\
\hline Hospitales & 30 \\
\hline Usuarios & 95 \\
\hline Portales Sanitarios & 14 \\
\hline Fundaciones & 8 \\
\hline Empresas sanitarias & 3 \\
\hline Instituciones & 7 \\
\hline Medios de información & 6 \\
\hline Congresos & 5 \\
\hline \multicolumn{2}{r|}{ Total } \\
\hline
\end{tabular}

Fuente: elaboración propia

\subsection{Análisis de datos}

Para la extracción de datos y análisis de las publicaciones de Twitter y Facebook, se utilizó ATLAS.ti software (versión 8.4, Scientific Software Development). A través de este software, se accedió a la interfaz de programación de aplicaciones (API) de transmisión de Twitter y Facebook, donde se extrajeron las publicaciones de ambas redes sociodigitales.

Por otro lado, se realizó una categorización abierta donde se crearon las categorías a medida que se realizaba el análisis de las diferentes publicaciones (Pizarro, 2000). Una vez que la codificación inicial se completó, los investigadores del presente estudio discutieron y finalizaron la codificación para completar el proceso de análisis. Para ello, se corroboró que las categorías escogidas eran adecuadas a las publicaciones analizadas en relación con el objetivo de investigación (Clarke y Braun, 2014). Para finalizar, se determinaron los nombres de las categorías en función del área donde se abordase el tema de la humanización, simbolizando así las ideas y referencias más destacadas en las publicaciones (Vaismoradi, Jones, Turunen, y Snelgrove, 2016).

\subsection{Consideraciones éticas}

Los tuits así como las entradas de Facebook analizadas para el presente estudio fueron extraídas de la API de Twitter y Facebook, a través del software ATLAS.ti. Todas

Revista de Comunicación y Salud, 2022, Vol. 12, 21-44 
ellas de perfiles públicos y de acceso abierto. Además, solo se hizo uso del contenido de las publicaciones y no de la información personal de la cuenta vinculada, por lo que no se hace uso de los datos personales de los usuarios que publicaron ese contenido.

\section{RESULTADOS}

En la actualidad, la difusión de la información a través de las redes sociodigitales se ha convertido en una herramienta esencial para la difusión del conocimiento. Es por ello, que en este trabajo se ha analizado la difusión que existe a día de hoy de la humanización en Twitter y Facebook, en la población general, en Estados Unidos, España, Italia, Colombia, Brasil, Portugal, Canadá y Francia.

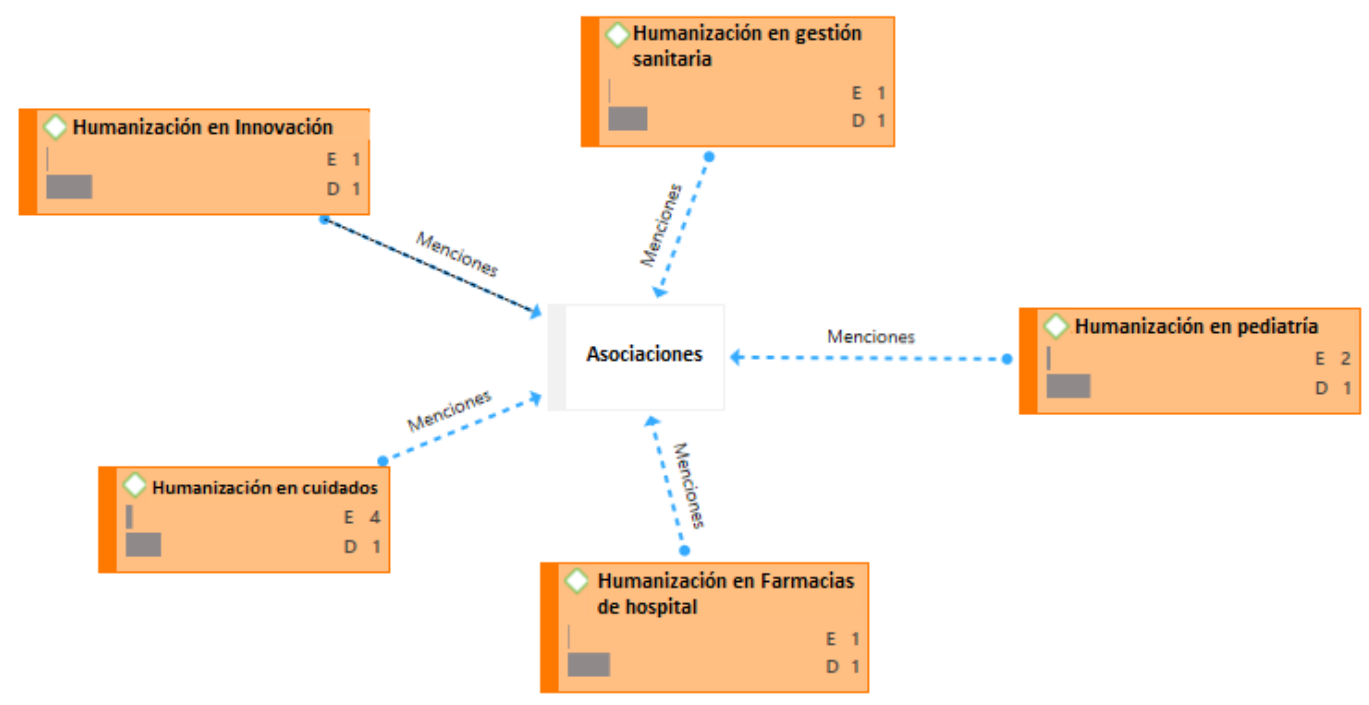

Figura 1. Publicaciones de asociaciones ${ }^{*} \mathrm{E}=$ Número de publicaciones. ${ }^{*} \mathrm{D}=$ Número de conexiones establecidas. Fuente: elaboración Propia

Tal y como se muestra en la Figura 1, se puede observar como la humanización en salud aparece en diferentes ámbitos sanitarios. En relación con las publicaciones analizadas de las diferentes asociaciones sanitarias, tales como asociaciones contra el cáncer, de pediatría, sobre enfermedades raras, entre otras. Los tuits más comentados tratan de la humanización en cuidados $(n=4)$, seguido de humanización en pediatría $(n=2)$, humanización en farmacia hospitalaria $(n=1)$, humanización en innovación $(n=1)$, y de humanización en gestión hospitalaria $(n=1)$, las asociaciones sanitarias muestran cómo el personal sanitario se implica con los pacientes y sus familiares, haciendo a la sociedad consciente de la importancia que tiene la humanización de los cuidados en los hospitales. 


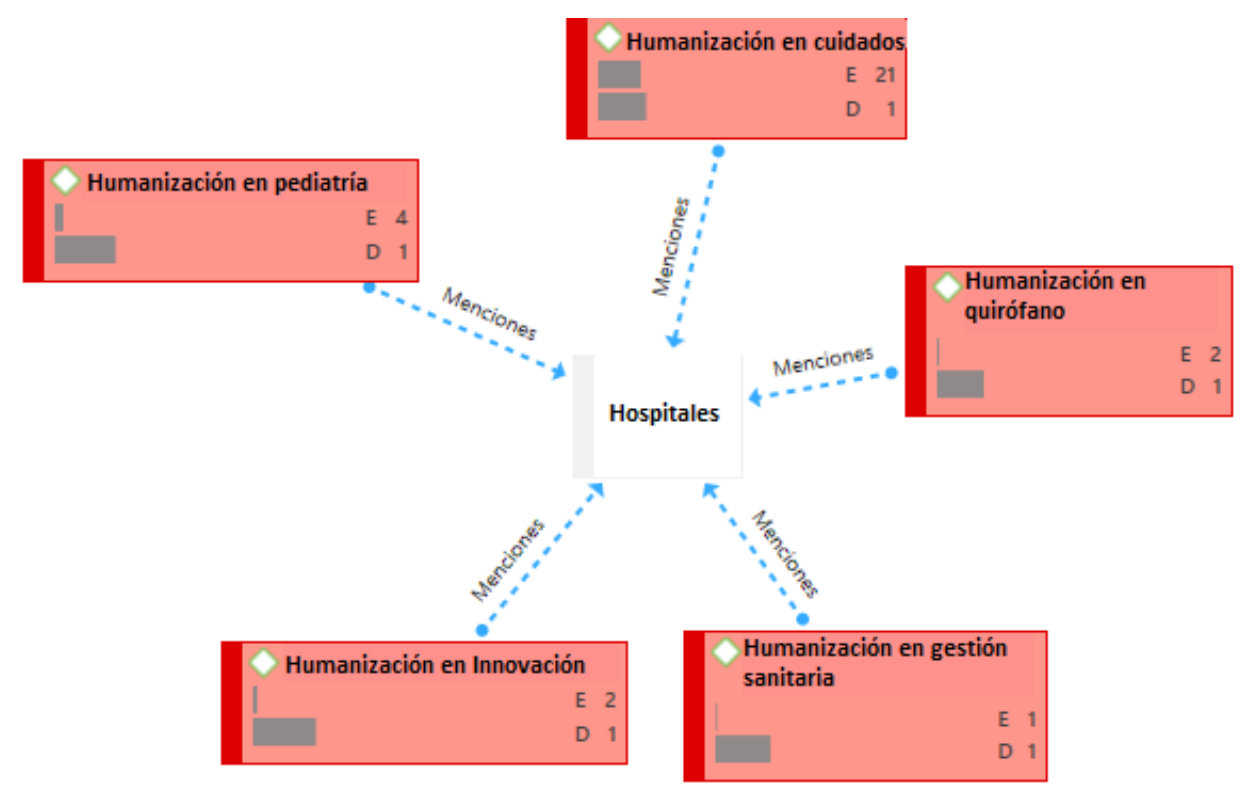

Figura 2. Publicaciones por parte de hospitales ${ }^{*} \mathrm{E}=$ Número de publicaciones.

${ }^{*} \mathrm{D}=$ Número de conexiones establecidas.

Fuente: elaboración Propia

Así bien, los hospitales son una de las principales fuentes de información y difusión de la humanización en el ámbito sanitario, es por ello, que se encuentran numerosos puntos de debate tanto en Facebook como Twitter, procedentes de hospitales, los cuales manifiestan la falta de humanización en todos los ámbitos. En su mayoría, muestran la importancia que posee la humanización en cuidados $(n=21)$ dentro del ámbito hospitalario donde el trato al paciente es fundamental, seguida de la humanización en pediatría $(n=4)$, de la humanización en quirófano $(n=2)$ y en innovación $(n=2)$, y la humanización en la gestión hospitalaria $(n=1)$. 


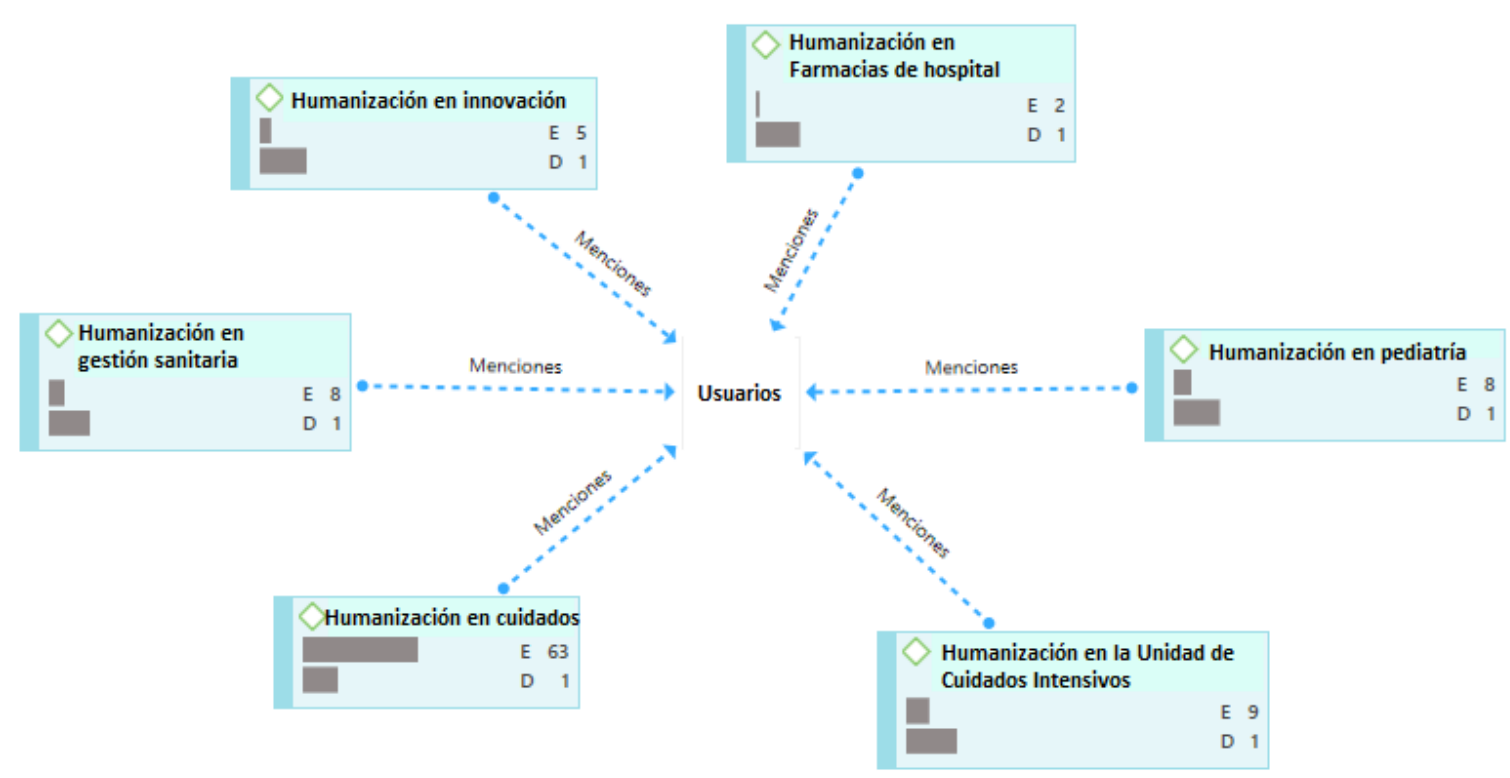

Figura 3. Temáticas abordadas por los usuarios de Twitter y Facebook

${ }^{*} \mathrm{E}=$ Número de publicaciones.

${ }^{*} \mathrm{D}=$ Número de conexiones establecidas.

Fuente: elaboración Propia

Como se puede observar en la Figura 3, los usuarios de Twitter o Facebook, son los que aportan una mayor cantidad de información y opiniones acerca de la humanización en el contexto sanitario, en mayor medida manifiestan sus opiniones acerca de la humanización en cuidados $(n=63)$, debido a que en la mayoría de ocasiones son los propios usuarios los que perciben esa humanización en los cuidados, seguido de la humanización en la Unidad de Cuidados Intensivos $(n=9)$, de la humanización en pediatría $(n=8)$ y en gestión hospitalaria $(n=8)$, de la humanización en Innovación sanitaria $(n=5)$, y de la humanización en Farmacias hospitalarias $(n=2)$. 
Análisis de la humanización en el ámbito sanitario en redes sociodigitales

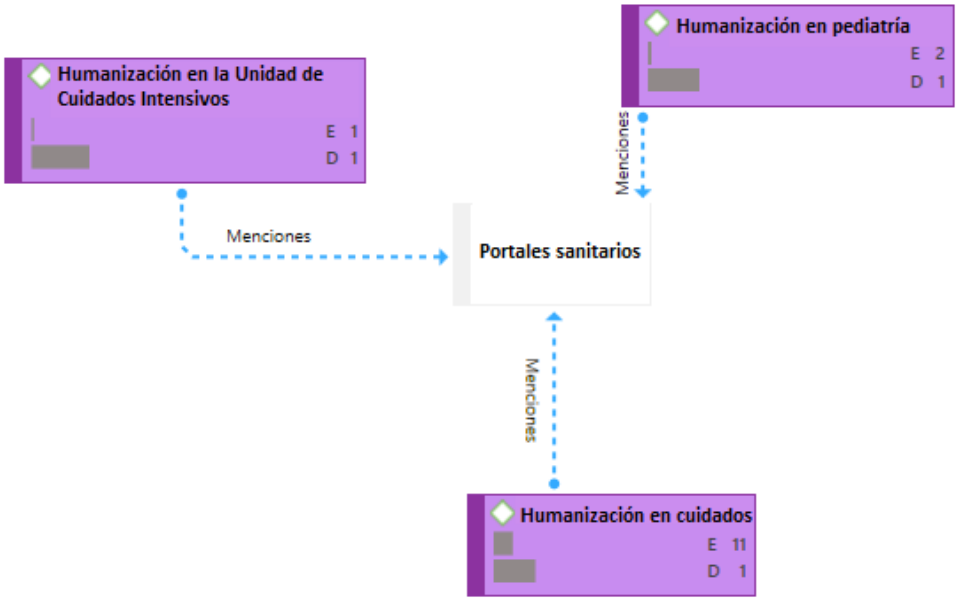

Figura 4. Temáticas abordadas por los portales sanitarios de Twitter y Facebook ${ }^{*} \mathrm{E}=$ Número de publicaciones.

${ }^{*} \mathrm{D}=$ Número de conexiones establecidas.

Fuente: elaboración Propia

Como se puede observar en la Figura 4, los portales sanitarios ofrecen diferentes opiniones acerca de la humanización sanitaria dentro de los cuidados $(n=11)$, así como en el área de pediatría $(n=2)$, y de la Unidad de cuidados intensivos $(n=1)$.

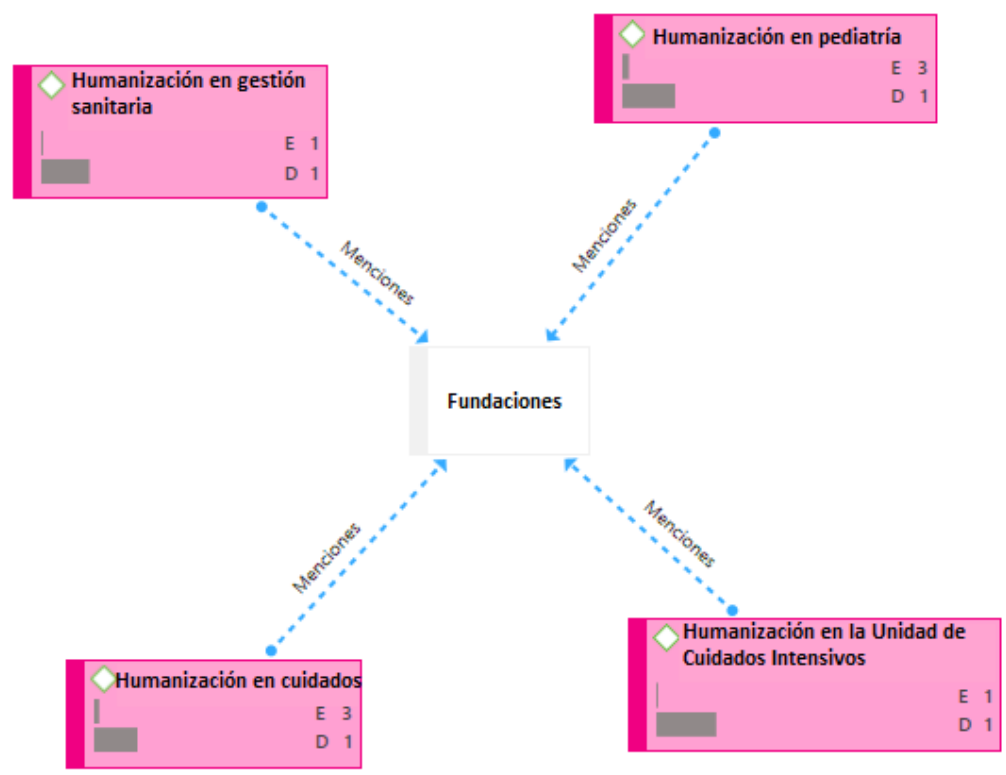

Figura 5. Publicaciones por parte de fundaciones ${ }^{*} \mathrm{E}=$ Número de publicaciones.

${ }^{*} \mathrm{D}=$ Número de conexiones establecidas.

Fuente: elaboración Propia 
Así mismo, son muchas las fundaciones que han comenzado a manifestar sus opiniones acerca de la humanización sanitaria, con el fin de expresar la adecuada humanización que comienza a dar cabida en los hospitales, así como las mejoras que necesita el sistema sanitario para garantizar la calidad asistencial sanitaria. Por ello, opinan en su mayoría sobre la humanización en cuidados $(n=3)$, la humanización en pediatría $(n=3)$, y la humanización en la unidad de cuidados intensivos $(n=1)$ y en gestión hospitalaria $(n=1)$.

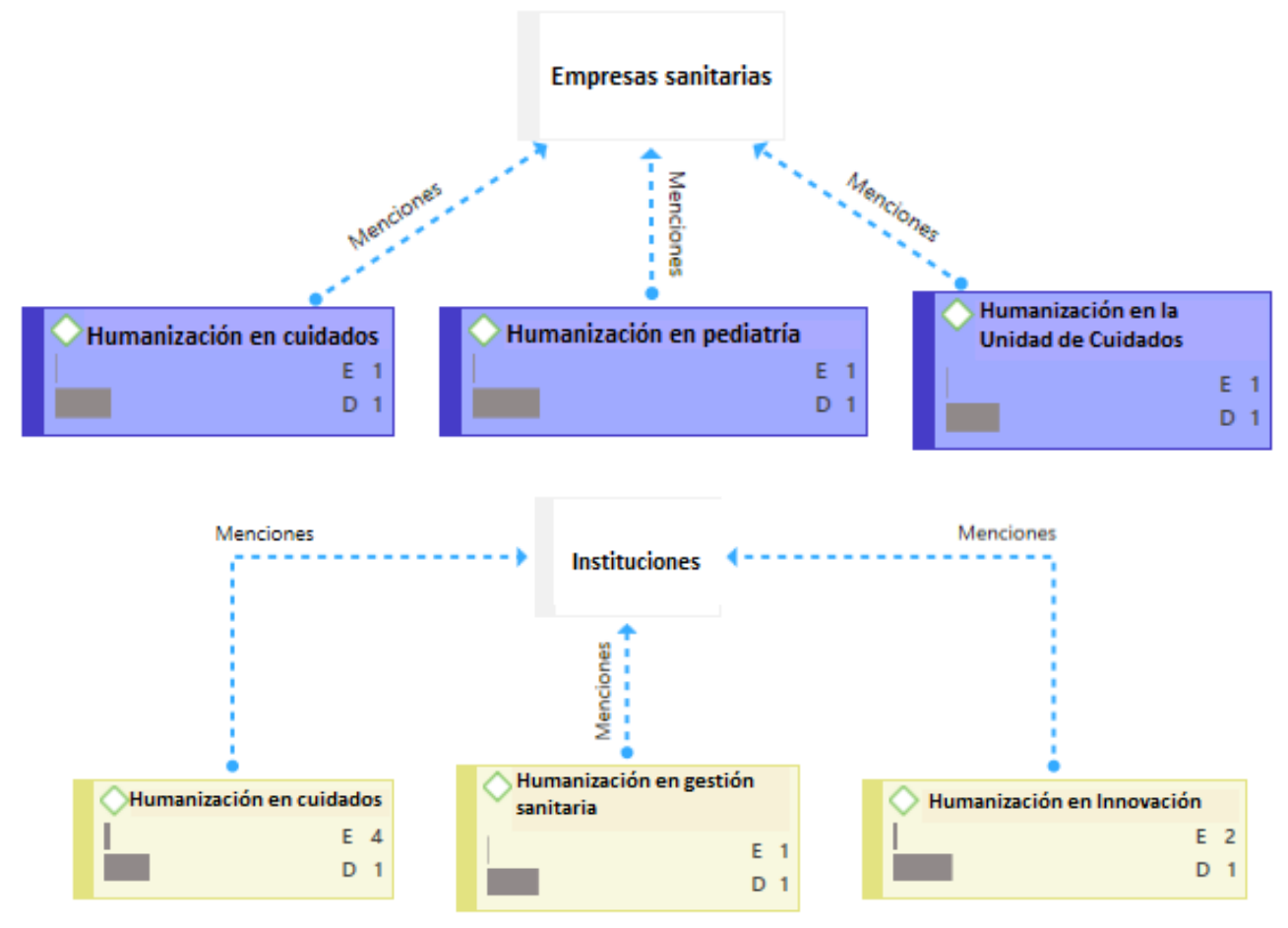

Figura 6. Publicaciones por parte de empresas sanitarias e instituciones ${ }^{*} \mathrm{E}=$ Número de publicaciones. ${ }^{*} \mathrm{D}=$ Número de conexiones establecidas.

Fuente: elaboración Propia

En la Figura 6, se analizan aquellas entradas procedentes de Instituciones y Empresas sanitarias. Se observa cómo las Empresas dedicadas a la sanidad comienzan a expresar sus opiniones sobre humanización en cuidados $(n=1)$, en pediatría $(n=1)$, y en la $\mathrm{UCl}(n=1)$. Por otro lado, encontramos las Instituciones, donde los temas de relevancia tratan la humanización en cuidados $(n=4)$, la humanización en Innovación $(n=2)$, y la humanización en gestión hospitalaria $(n=1)$. 


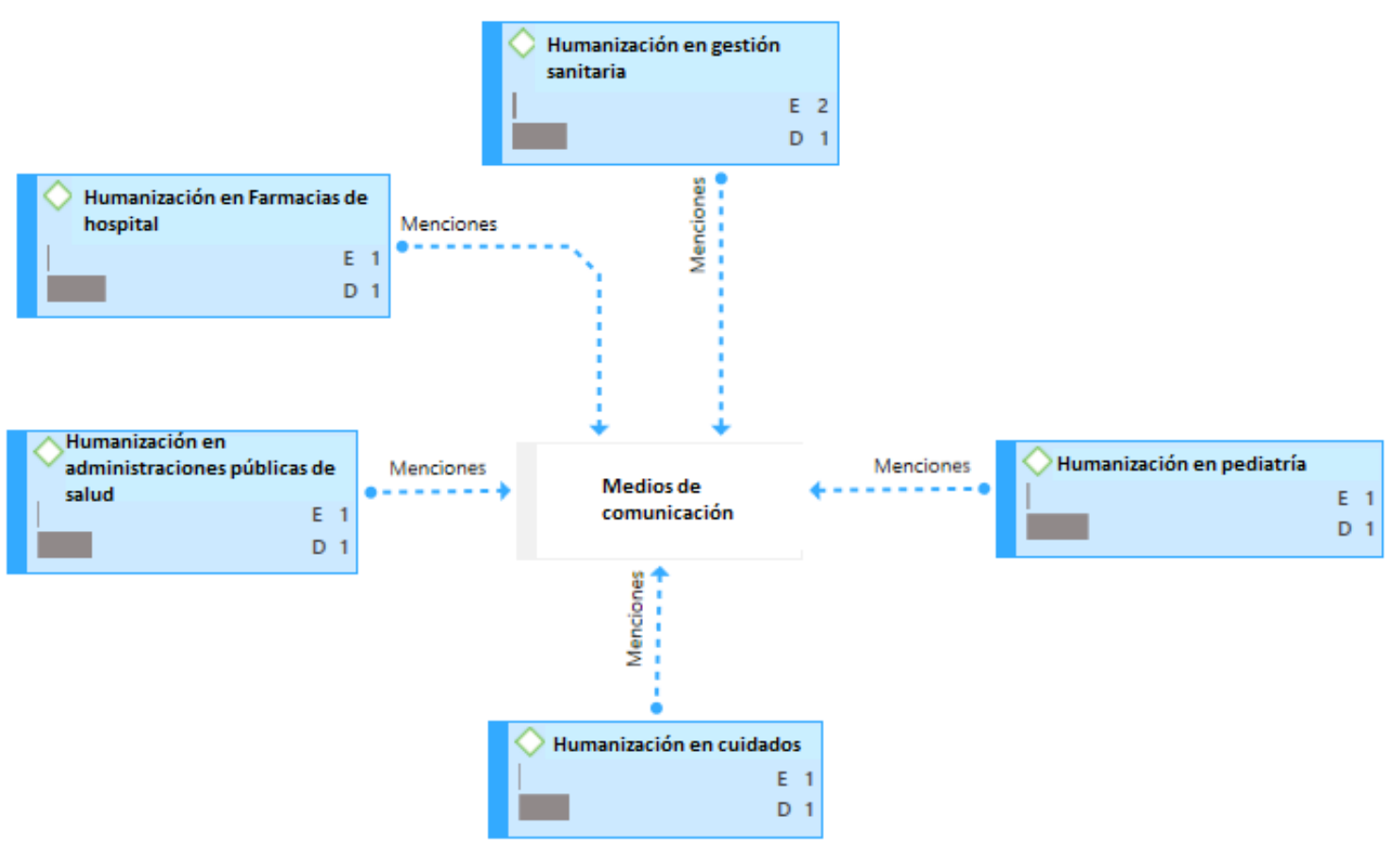

Figura 7. Publicaciones por parte de medios de información

${ }^{\star} \mathrm{E}=$ Número de publicaciones.

${ }^{*} \mathrm{D}=$ Número de conexiones establecidas.

Fuente: elaboración Propia

Tal y como se muestra en la Figura 8, los medios de divulgación en la actualidad se han hecho eco de la importancia de la humanización en la sanidad, es por ello que diversas de sus entradas están destinadas a divulgar información de esta. Estos medios difunden noticias acerca de la humanización en gestión $(n=2)$, en cuidados $(n=1)$, en las administraciones públicas (1), farmacias hospitalarias (1) y pediatría (1). 


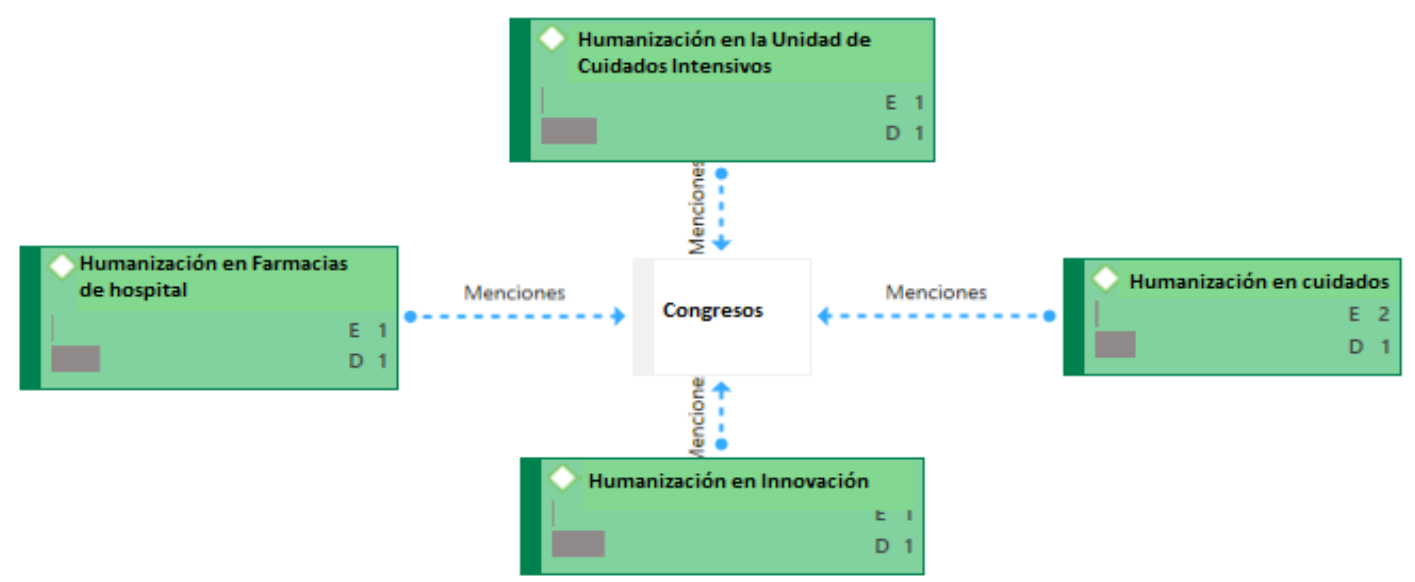

Figura 8. Publicaciones en congresos

${ }^{*} \mathrm{E}=$ Número de publicaciones.

${ }^{*} \mathrm{D}=$ Número de conexiones establecidas.

Fuente: elaboración Propia

Por último, encontramos diversos Tuits de congresos dedicados a la temática de la humanización en sanidad, analizando así la red social de los mismos, los cuales tratan temas acerca de la humanización en cuidados $(n=2)$, la humanización en la unidad de cuidados intensivos $(n=1)$, la humanización en farmacias hospitalarias $(n=1)$ y de la humanización en innovación $(n=1)$.

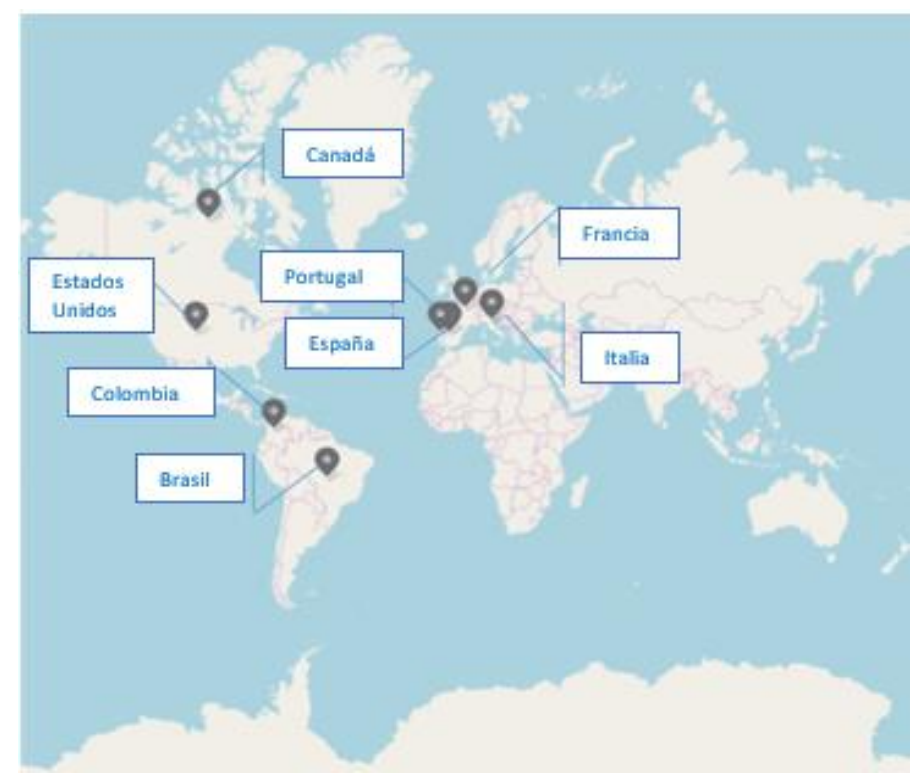

Figura 9. Distribución de la humanización por países

Fuente: elaboración Propia 
La información en redes sociodigitales se difunde a través de diferentes países gracias a las nuevas tecnologías, por lo que estamos interconectados. Es por ello, que se ha analizado el debate sobre la humanización sanitaria en diferentes países, entre los que se encuentran España $(n=127)$, Brasil $(n=10)$, Colombia $(n=7)$, Italia $(n=10)$, Francia $(n=10)$, Estados Unidos $(n=5)$, Canadá $(n=3)$, y Portugal $(n=2)$, en un menor porcentaje. Estos mensajes se centraron en la difusión de humanización en el ámbito sanitario con el fin de otorgar importancia a la humanización en los hospitales. Además, se comparte a través de las redes sociodigitales analizadas la labor que realizan los profesionales sanitarios, donde la humanización adquiere un espacio fundamental en todas las áreas de los hospitales.

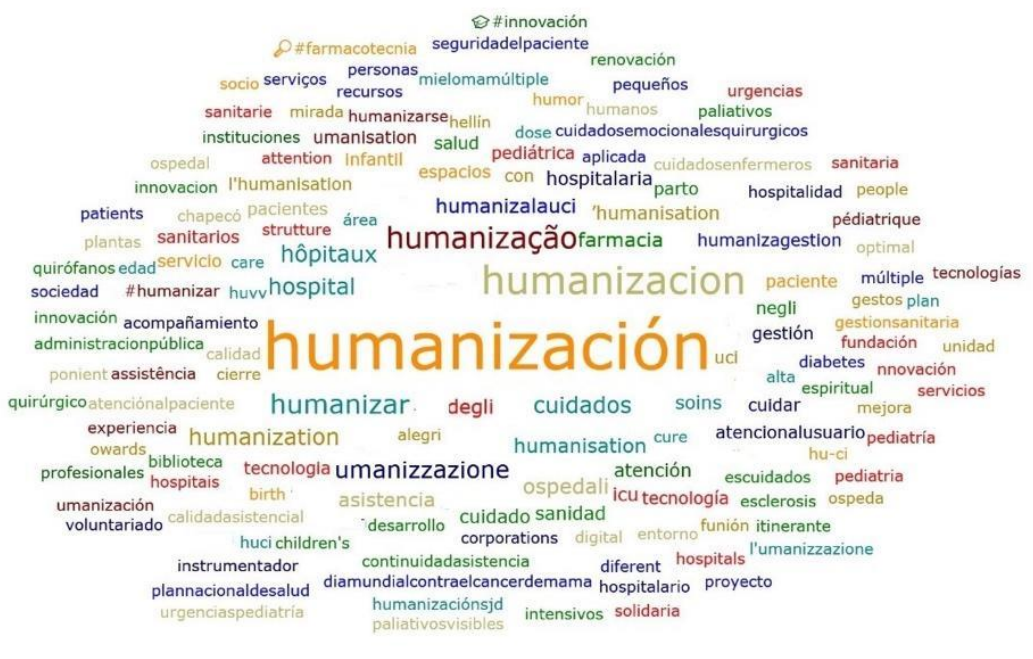

Figura 10. Nube de palabras Fuente: elaboración Propia

En esta misma línea, se corrobora la importancia de las palabras clave en los trabajos científicos, ya que facilita visualizar los términos más populares en las redes sociodigitales. Por ello, en la Figura 10 se puede observar cómo el tamaño de los diferentes conceptos que aparecen en la nube de palabras representan la relevancia que tiene cada una de las palabras clave relacionadas con el número de tuits y entradas de Facebook que las contienen. Las palabras que se pueden observar en la nube de palabras están relacionadas con el término de "humanización de la salud" en los diferentes idiomas utilizados para el presente trabajo.

\section{DISCUSIÓN}

El incremento del uso de las redes sociodigitales, se ha convertido en un fenómeno común en los últimos años, ocasionando un gran cambio en la forma de comunicarse y socializarse de las personas. Así mismo, se percibe que las personas pasan gran parte 
de su tiempo libre navegando por las redes sociodigitales, lo que facilita el intercambio de conocimientos, información e ideas (Kakushi y Évora, 2016).

Las redes sociodigitales ayudan en múltiples de ocasiones a poner de manifiesto los déficits que sufre la sanidad pública. Así como, informan sobre diversas temáticas que a día de hoy no han sido estudiadas en profundidad en trabajos de investigación como es el tema de la humanización en sanidad, donde encuentran apoyo social de diferentes usuarios (Zhang y Xiang, 2019). Por ello, el presente trabajo ha indagado sobre el impacto que ha tenido la humanización sanitaria en las redes sociodigitales.

Han sido diferentes las fuentes que expresan opiniones sobre la humanización hospitalaria desde los propios hospitales, congresos dirigidos exclusivamente a esta temática, pacientes, familiares, fundaciones, asociaciones, sindicatos, entre otros. De forma general, opinan sobre la importancia que tiene en los últimos años la humanización en la sanidad (Catapan et al., 2019; Pérez-Fuentes et al., 2019a). Es imprescindible mejorar la atención a los pacientes para lograr así una atención de calidad y en consecuencia mejorar los sistemas sanitarios (Carli et al., 2018).

De forma común, la mayoría de entradas tanto en Facebook como Twitter, hablan de la importancia de la humanización en cuidados y el trato dirigido tanto al paciente como a sus familiares y a los propios profesionales sanitarios, siendo imprescindibles en la humanización en cuidados dentro del sistema sanitario (Pérez-Fuentes et al., 2019). Así mismo, reivindican desde todos los ámbitos sociales la poca visibilidad que se le da a la humanización en cuidados, cayendo en el error de creer que no es de relevancia para el éxito en la sanidad (Calegari et al., 2015).

Por otro lado, se observa la preocupación y el interés que genera la humanización en pediatría a nivel social, por ello, es necesario hacer hincapié en la importancia que tiene la humanización dentro del área pediátrica, donde las redes sociodigitales sirven para que el público, los pacientes y los profesionales sanitarios expresen su punto de vista e intercambien información sobre los problemas que surgen en el sistema sanitario y les permita mejorar los resultados e intervenir para solventar los problemas que se les plantean (Chan y Leung, 2018; Moorhead et al., 2013).

Así mismo, se muestra un incipiente interés por parte de los usuarios de las redes sociodigitales acerca de la humanización en la Unidad de Cuidados Intensivos, manifestando la relevancia que tiene el cuidado del paciente en esta área, por lo que se hace indispensable seguir trabajando la humanización en la UCI para sensibilizar a los profesionales sanitarios, garantizando un entorno más familiar y acogedor donde la familia pueda ser partícipe y colaborar con su recuperación (Camelo, 2012; Luiz et al., 2017).

Para finalizar, se muestra como la humanización es de interés en diferentes países, sobre todo en Brasil donde se están llevando a cabo políticas humanizadoras en el ámbito sanitario, donde las evidentes diferencias sociales han determinado la necesidad de generar programas de humanización con la finalidad de garantizar la 
igualdad de oportunidades de atención y revisión de toda la población (Tripodi et al., 2019).

De los resultados expuestos anteriormente, se pueden derivar futuras líneas de investigación. Pudiendo ser de interés aumentar el número de entradas en Facebook y Twitter analizadas, de esta manera recogeremos resultados más amplios de dicha investigación. Así mismo, se podría ampliar el contexto al que está dirigido, analizando diferentes ámbitos profesionales como el ámbito educativo, judicial, entre otros.

\section{CONCLUSIONES}

De forma general, se muestra cómo las redes sociodigitales son de vital importancia para la comunicación e intercambio de información y conocimientos sobre la humanización, una temática que comienza a suscitar interés a nivel social dentro del ámbito sanitario, por tanto, las redes sociodigitales son esenciales para realizar programas de intervención para la mejora de la calidad de los sistemas sanitarios y en consecuencia mejorar la atención sanitaria dirigida a pacientes, familiares y profesionales de la salud.

En base a los resultados expuestos, se hace imprescindible que los hospitales, así como los portales sanitarios, se impliquen e interactúen más en las redes sociodigitales, con el objetivo de informar al resto de usuarios de Facebook o Twitter de la importancia de la humanización en el ámbito sanitario, ya que, estas son unas de las categorías analizadas que menos publicaciones se han hallado, siendo ambas propicias para la divulgación de la humanización.

El presente trabajo trata de reflejar la importancia que han adquirido las redes sociodigitales como Facebook y Twitter, para reivindicar los déficits que tiene la sanidad actualmente, así como reconocer el mérito que supone el avance de la humanización en los cuidados del paciente. No obstante, se debe seguir poniendo de manifiesto la falta de información existente sobre humanización en el ámbito de la investigación científica, por lo cual, se debe seguir haciendo hincapié en que no solo se debe exponer esta información en redes, sino también investigar y hacer conocer dicha información en artículos científicos.

\section{REFERENCIAS}

Amoah, P. A., Edusei, J., y Amuzu, D. (2018). Social Networks and Health: Understanding the Nuances of Healthcare Access between Urban and Rural Populations. International Journal of Environmental Research and Public Health, 15(5), 973. https://doi.org/10.3390/ijerph15050973

Anwar, M. M., Liu, C., y Li, J. (2019). Discovering and tracking query oriented active online social groups in dynamic information network. World Wide Web, 22(4), 18191854. https://doi.org/10.1007/s11280-018-0627-5. 
Bandura, A., O'Leary, A., Taylor, C. B., Gauthier, J., \& Gossard, D. (1987). Perceived self-efficacy and pain control: Opioid and nonopioid mechanisms. Journal of Personality and Social Psychology, 53(3), 563-571. https://doi.org/10.1037/0022$\underline{3514.53 .3 .563}$

Bartosiewicz, A., y Januszewicz, P. (2019). Readiness of Polish Nurses for Prescribing and the Level of Professional Burnout. International Journal of Environmental Research and Public Health, 16(1), 35. https://doi.org/10.3390/ijerph16010035

Bendayan, R., y Blanca, M. J. (2019). Spanish version of the Facebook Intrusion Questionnaire (FIQ-S). $\quad$ Psicothema, 31(2), 204-209. https://doi.org/10.7334/psicothema2018.301

Borbasi, S., Galvin, K. T., Adams, T., Todres, L., Farrelly, B. (2012). Demonstration of the usefulness of a theoretical framework for humanising care with reference to a residential aged care service in Australia. Journal of Clinical Nursing, 22(5-6), 881889. https://doi.org/10.1111/j.1365-2702.2012.04256.x

Calegari, R., Massarollo, M. C., y Dos Santos, M. J. (2015). Humanization of health care in the perception of nurses and physicians of a private hospital. Revista da Escola de Enfermagem da USP, 49(2), 41-46. https://doi.org/10.1590/S0080$\underline{623420150000800006}$

Camelo, S. H. (2012). Professional competences of nurse to work in Intensive Care Units: an integrative review. Revista Latino-Americana de Enfermagem, 20(1), 192200. https://doi.org/10.1590/S0104-11692012000100025

Carli, B. S., Ubessi, L. D., Pettenon, M. K., Righi, L. B., Jardim, V. M. R., y Stumm, E. M. F. (2018). The humanization theme in intensive care in health studies. Revista de Pesquisa: Cuidado é Fundamental, 10(2), 326-333. https://doi.org/10.9789/21755361.2018.v10i2.326-333

Carver, C. S., y Scheier, M. F. (2014). Dispositional optimism. Trends in Cognitive Sciences, 18(6), 293-299. https://doi.org/10.1016/j.tics.2014.02.003

Catapan, S. C., de Oliveira, W. F., y Rotta, T. M. (2019). Palhaçoterapia em ambiente hospitalar: Uma revisão de literatura. Ciencia e Saude Coletiva, 24(9), 3417-3429. https://doi.org/10.1590/1413-81232018249.22832017

Chan, W. S., y Leung, A. Y. (2018). Use of Social Network Sites for Communication Among Health Professionals: Systematic Review. Journal of Medical Internet Research, 20(3), e117. https://doi.org/10.2196/jmir.8382

Di Fabio, A., Palazzeschi, L., Bucci, O., Guazzini, A., Burgassi, C., y Pesce, E. (2018). Personality Traits and Positive Resources of Workers for Sustainable Development: 
Análisis de la humanización en el ámbito sanitario en redes sociodigitales

Is Emotional Intelligence a Mediator for Optimism and Hope? Sustainability, 10(10), 3422, https://doi.org/10.3390/su10103422

Erfani, S. S., y Abedin, B. (2018). Impacts of the use of social network sites on users' psychological well-being: A systematic review. Journal of the Association for Information Science and Technology, 69, 900-912. https://doi.org/10.1002/asi.24015

García-Rodríguez, A., Gutiérrez-Bedmar, M., Bellón-Saameño, J. Á., Muñoz-Bravo, C. y Fernández-Crehuet, J. N. (2015). Psychosocial stress environment and health workers in public health: Differences between primary and hospital care. Atención Primaria, 47(6), 359-366. https://doi.org/10.1016/..aprim.2014.09.003

García-Salido, A., Heras, G., y Serrano, A. (2019). Narrative review of pediatric critical care humanization: Where we are? Medicina Intensiva, 43(5), 290-298.

Griffiths, F., Cave, J., Boardman, F., Ren, J., Pawlikowska, T., Ball, R., Clarke, A., y Cohen, A. (2012). Social Networks-The Future for Health Care Delivery." Social Science and Medicine 75(12), 2233-2241. https://doi.org/10.1016/i.socscimed.2012.08.023

Griffiths, F., Dobermann, T., Cave, J. A., Thorogood, M., Johnson, S., Salamatian, K. y Goudge, J. (2015). The Impact of Online Social Networks on Health and Health Systems: A Scoping Review and Case Studies. Policy \& Internet, 7(4), 473-496. https://doi.org/10.1002/poi3.97

Howick, J., Steinkopf, L., Ulyte, A., Roberts, N., y Meissner, K. (2017). How empathic is your healthcare practitioner? A systematic review and meta-analysis of patient surveys. BMC Medical Education, 17(1), 136. https://doi.org/10.1186/s12909-017$\underline{0967-3}$

Kakushi, L. E., y Évora, Y. D. (2016). Social networking in nursing education: integrative literature review. Revista Latino-Americana de Enfermagem, 24, e2709. https://doi.org/10.1590/1518-8345.1055.2709

Klimova, B., y Pikhart, M. (2020). Cognitive and Applied Linguistics Aspects of Using Social Media: The Impact of the Use of Facebook on Developing Writing Skills in Learning English as a Foreign Language. European Journal of Investigation in Health, Psychology and Education, 10(1), 10. https://doi.org/10.3390/ejihpe10010010

Lown, B. A., Mclntosh, S., Gaines, M. E., McGuinn, K., y Hatem, D. S. (2016). Integrating compassionate, collaborative care (the "Triple C") into health professional education to advance the triple aim of health care. Academic Medicine, 91(3), 310316. https://doi.org/10.1097/ACM.0000000000001077 . 
Análisis de la humanización en el ámbito sanitario en redes sociodigitales

Luiz, F. F., Caregnato, R. C. A., y Costa, M. R. (2017). Humanization in the Intensive Care: perception of family and healthcare professionals. Revista Brasileira Enfermegen, 70(5), 1040-1047. https://doi.org/10.1590/0034-7167-2016-0281

Mehdipour, Y. (2019). Medical students' life and virtual social networks a guide for educational managers and parents. Journal of Family Medicine and Primary Care, 8(8), 2573-2576. https://doi.org/10.4103/ifmpc.jfmpc 33619

Molero, M. M., Pérez-Fuentes M. C., Gázquez, J. J., y Barragán, A. B. (2018a). Burnout in Health Professionals According to Their Self-Esteem, Social Support and Empathy Profile. Frontiers Psychology, 20(9), 424. https://doi.org/10.3389/fpsyg.2018.00424

Molero, M. M., Pérez-Fuentes, M. C., Gázquez, J. J., Simón, M. M., y Martos, A. (2018b). Burnout Risk and Protection Factors in Certified Nursing Aides. International Journal of Environmental Research and Public Health, 15(6), 1116. https://doi.org/10.3390/ijerph15061116

Moorhead, S. A., Hazlett, D. E., Harrison, L., Carroll, J. K., Irwin, A. y Hoving, C. (2013). A new dimension of health care: systematic review of the uses, benefits, and limitations of social media for health communication. Journal of Medical Internet Research, 15(4), e85. https://doi.org/10.2196/imir.1933.

Nora, C. R. y Junges, J. R. (2013). Política de humanização na atenção básica: revisão sistemática [Humanization policy in primary health care: a systematic review]. Revista de Saude Publica, 47(6), 1186-1200. https://doi.org/10.1590/s0034$\underline{8910.2013047004581}$

Penni, J. (2017). The future of online social networks (OSN): A measurement analysis using social media tools and application. Telematics and Informatics, 34(5), 498-517. https://doi.org/10.1016/j.tele.2016.10.009 .

Pérez-Fuentes, M., Molero, M., Herrera-Peco, I., Oropesa, N. F. y Gázquez, J. (2019a). Propuesta de un Modelo de Humanización basado en las Competencias Personales: Modelo HUMAS. European Journal of Health Research, 5(1), 63-77. https://doi.org/10.30552/ejhr.v5i1.173

Pérez-Fuentes, M. C., Herrera-Peco, I., Molero, M. M., Oropesa, N. F., Ayuso-Murillo, D. y Gázquez, J. J. (2019b). The Development and Validation of the Healthcare Professional Humanization Scale (HUMAS) for Nursing. International Journal of Environmental Research and Public Health, 16(20), 3999. https://doi.org/10.3390/ijerph16203999

Pérez-Fuentes, M. C., Molero, M. M., Gázquez, J. J. y Oropesa, N. F. (2018). The Role of Emotional Intelligence in Engagement in Nurses. International Journal of Environmental Research and Public Health, 15(9), 1915. https://doi.org/10.3390/ijerph15091915 
Análisis de la humanización en el ámbito sanitario en redes sociodigitales

Pérez-Fuentes, M. C., Molero, M. M., y Gázquez, J. J. (2019d). Explanatory Value of General SelfEfficacy, Empathy and Emotional Intelligence in Overall Self-Esteem of Healthcare Professionals. Social Work in Public Health, 34(4), 318-329. https://doi.org/10.1080/19371918.2019.1606752

Pérez-Fuentes, M. C., Molero-Jurado, M. M., Gázquez-Linares, J. J. y Simón-Márquez, M. M. (2019c). Analysis of Burnout Predictors in Nursing: Risk and Protective Psychological Factors. The European Journal of Psychology Applied to Legal Context, 11(1), 33-40. https://doi.org/10.5093/ejpalc2018a13

Perkins, J. M., Subramanian, S. V. y Christakis, N. A. (2015). Social networks and health: a systematic review of sociocentric network studies in low- and middle-income countries. Social Science \& Medicine, 125, 60-78. https://doi.org/10.1016/j.socscimed.2014.08.019

Pizarro, A. (2000). El análisis de estudios cualitativo. Atención Primaria, 25(1), 42-46.

Scanfeld, D., Scanfeld, V. y Larson, E. L. (2010). Dissemination of Health Information Through Social Networks: Twitter and Antibiotics. American Journal of Infection Control, 38(3), 182-188. https://doi.org/10.1016/j.ajic.2009.11.004 .

Segerstrom, S. C., Carver, C. S., Scheier, M. F. Optimism. In The Happy Mind: Cognitive Contributions to Well-Being; Robinson, M. D., Eid, M., (Eds), Springer International Publishing: Cham, Switzerland, 2017, pp. 195-212.

Seligman, M. E., Schulman, P. y Tryon, A. M. (2007). Group prevention of depression and anxiety symptoms. Behaviour Research and Therapy, 45(6), 1111-1126.

Soriano, J. G., Pérez-Fuentes, M. C., Molero, M. M., Tortosa, B. M., y González, A. (2019). Beneficios de las intervenciones psicológicas en relación al estrés y ansiedad: Revisión sistemática y metaanálisis. European Journal of Education and Psychology, 12(2), 191-206. https://doi.org/10.30552/ejep.v12i2.283

Tripodi, M., Siano, M. A., Mandato, C. De Anseris, A. G. E., Quitadamo, P., Nuzio, S. G., Siani, P., y Vajro, P. (2019). Humanization interventions in general pediatric wards: a systematic review. European Journal of Pediatrics, 178(5), 607-622. https://doi.org/10.1007/s00431-019-03370-3

Wersebe, H., Lieb, R., Meyer, A. H., Hofer, P., y Gloster, A.T. (2018). The link between stress, well-being, and psychological flexibility during an Acceptance and Commitment Therapy self-help intervention. International Journal of Clinical and Health Psychology, 18(1), 60-68. https://doi.org/10.1016/j.ijchp.2017.09.002 
Análisis de la humanización en el ámbito sanitario en redes sociodigitales

Zhang, S., y Xiang, W. (2019). Income gradient in health-related quality of life - the role of social networking time. International Journal for Equity in Health, 18(1), 44. https://doi.org/10.1186/s12939-019-0942-1

Ziebland, S., y Wyke, S. (2012). Health and Illness in a Connected World: How Might Sharing Experiences on the Internet Affect People's Health? The Milbank Quarterly, 90(2), 219-49. https://doi.org/10.1111/j.1468-0009.2012.00662.x

Clarke, V., y Braun, V. (2014). Thematic analysis. In A.C. Michalos (Ed.), Encyclopedia of quality of life and well-being research (pp. 6626-6628). Springer.

Vaismoradi, M., Jones, J., Turunen, H., y Snelgrove, S. (2016). Theme development in qualitative content analysis and thematic analysis. Journal of Nursing and Practice, 6 , $100-110$.

García Del Castillo, J. A., García Del Castillo-López, Á., Días, P. C., \& García-Castillo, F. (2020). Social networks as tools for the prevention and promotion of health among youth. Psicologia, Reflexao e Critica, 33(1), 13. https://doi.org/10.1186/s41155-020$\underline{00150-z}$

\section{AUTORES}

\section{María del Carmen Pérez-Fuentes}

Profesora Titular de Universidad y Directora del Grupo SEJ-581 Investigación en el ámbito educativo y de la salud de la Universidad de Almería. Ha sido Directora de la Unidad de Calidad, del Secretariado de la Universidad de Mayores, y Secretaria del Departamento de Psicología. Actualmente, Coordinadora del Máster Universitario en Intervención en Convivencia Escolar, del Máster en Demencias y Enfermedad de Alzheimer, del Máster en Salud Pública y promoción de la Salud, así como, de varios Expertos Universitarios. Es directora de la European Journal of Investigation in Health, Psychology and Education, incluida en el Emerging Sources Citation Index, y forma parte del Consejo Editorial de varias publicaciones científicas incluidas en diferentes bases de datos internacionales (European Journal of Education and Psychology, Psicothema, Frontiers in Psychology, International Journal of Clinical and Health Psychology).

Autora de más de 75 artículos incluidos en el JCR y múltiples artículos científicos en otras bases de datos (ESCl, SCOPUS, etc.), editora de más de 60 libros, y autora de casi 200 capítulos de libro, de múltiple material docente y de aportaciones a congreso y ponencias. Investigadora Principal de varios proyectos de $\mathrm{I}+\mathrm{d}+\mathrm{i}$, autonómicos y nacionales, Proyectos de Innovación Docente, beneficiaria de una Beca Salvador de Madariaga, así como, miembro del equipo con propiedad intelectual del Programa de Estimulación Cognitiva e Inteligencia Emocional, y de EMOCOVID.

Orcid ID: https://orcid.org/0000-0001-5950-5175

Google Scholar: https://scholar.google.es/citations?user=M9olT08AAAAJ\&hl=es 


\section{María del Mar Molero Jurado}

Profesora Titular de Universidad y directora del Grupo de Investigación SEJ-473. Respecto al ámbito de la investigación, ha publicado un total de 98 artículos, 50 de ellos recogidos en el Journal Citation Reports del Social Science Citation Index de Claritive Analytics. En cuanto a los otros artículos, han sido publicados en revistas que cumplen con los criterios de bases de datos tales como: Psycinfo; Eric, Ebsco Host, Ulrichs' Periodicals; DOAJ; Latindex; Scopus; DICE; así como el in-RECS. Ha publicado 84 monografías, así como 58 actas de congresos, 48 de ellas de carácter internacional, y un total de 191 capítulos de libro. Ha participado en un proyecto de excelencia de la junta de Andalucía y forma parte del equipo investigador de un proyecto I+D sobre el uso de la realidad aumentada para la detección e intervención en violencia entre iguales y consumo de sustancias en estudiantes de educación secundaria. Posee una solicitud de marcas/patentes y, en cuanto a ponencias y aportaciones a congresos y jornadas ser refiere, han sido 230 las aportaciones. En cuanto a otros méritos de investigación, destaca: la pertenencia a comités de organización de diferentes congresos nacionales e internacionales, miembro de 3 comités de edición/redacción de revistas científicas, European Journal of Investigation in Health, Psychology and Education, Directora de la European Journal of Health Research, y Sustainability. De igual modo, ha publicado material docente y ha participado en dos proyectos de innovación docente.

Orcid ID: https://orcid.org/0000-0001-9187-1474

Google Scholar: https://scholar.google.es/citations?user=e4DbyywAAAAJ\&hl=es

\section{José Jesús Gázquez Linares}

Catedrático de Universidad en Psicología Evolutiva y de la Educación. Director de la UNED en Almería. Ha sido Director del Secretariado de la Universidad de Mayores, y Director del Departamento de Psicología de la UAL. Director de la European Journal of Education and Psychology, forma parte del Consejo Editorial de varias publicaciones científicas incluidas en diferentes bases de datos internacionales. Autor de múltiples artículos científicos (JCR, ESCI, SCOPUS, etc.), libros y capítulos de libro, de múltiple material docente y de aportaciones a congreso y ponencias. Investigador Principal de varios proyectos de $\mathrm{I}+\mathrm{d}+\mathrm{i}$, Nacionales y de Excelencia de ámbito autonómico, y miembro del equipo con propiedad intelectual del Programa de Estimulación Cognitiva e Inteligencia Emocional. Director de múltiples tesis doctorales, trabajos fin de máster, de carrera y grado. Organizador y presidente de múltiples congresos desde 2005 relacionados con educación, psicología y salud.

Orcid ID: https://orcid.org/0000-0002-6969-1781

Google Scholar: https://scholar.google.es/citations?user=3jddYpAAAAAJ\&hl=es

\section{Ana Belén Barragán Martín}

Profesora Ayudante Doctor de la Universidad de Almería. Respecto al ámbito de la investigación, ha publicado un total de 37 artículos que han sido publicados en revistas que cumplen con los criterios de bases de datos tales como: Psycinfo; Eric, Ebsco Host, Ulrichs' Periodicals; DOAJ; Latindex; DICE; así como el in- RECS. Ha publicado 66 monografías, así como 52 actas de congresos, todas ellas de carácter internacional, y un total de 134 capítulos de libro. En cuanto a ponencias y aportaciones a congresos y jornadas se refiere, han sido 132 las aportaciones. En la actualidad, forma parte del 
equipo trabajo de un proyecto $\mathrm{I}+\mathrm{D}$ sobre el uso de la realidad aumentada para la detección e intervención en violencia entre iguales y consumo de sustancias en estudiantes de educación secundaria. Respecto a otros méritos de investigación, destaca: la pertenencia a comités de organización de diferentes congresos internacionales, miembro de 2 comités de edición/redacción de revistas científicas, la European Journal of Health Research y la European Journal of Child Development, Education and Psychopathology. La participación que ha tenido en cursos y congresos ha sido numerosa.

Orcid ID: https://orcid.org/0000-0001-8936-0245

Google Scholar: https://scholar.google.es/citations?hl=es\&user= B MIOAAAAAJ

\section{África Martos Martínez}

Profesora Ayudante Doctor. Respecto al ámbito de la investigación, ha publicado un total de 40 artículos que han sido publicados en revistas que cumplen con los criterios de bases de datos tales como: Psycinfo; Eric, Ebsco Host, Ulrichs' Periodicals; DOAJ; Latindex; DICE; así como el in- RECS. Ha publicado 66 monografías, así como 52 actas de congresos, todas ellas de carácter internacional, y un total de 134 capítulos de libro. En cuanto a ponencias y aportaciones a congresos y jornadas se refiere, han sido 132 las aportaciones. En la actualidad, forma parte del equipo trabajo de un proyecto I+D sobre el uso de la realidad aumentada para la detección e intervención en violencia entre iguales y consumo de sustancias en estudiantes de educación secundaria. Respecto a otros méritos de investigación, destaca: la pertenencia a comités de organización de diferentes congresos internacionales, miembro de 2 comités de edición/redacción de revistas científicas, la European Journal of Health Research y la European Journal of Child Development, Education and Psychopathology. La participación que ha tenido en cursos y congresos ha sido numerosa.

Orcid ID: https://orcid.org/0000-0002-6794-3906

\section{María del Mar Simón Márquez}

Doctora en Educación. Respecto al ámbito de la investigación, ha publicado un total de 10 artículos que han sido publicados en revistas que cumplen con los criterios de bases de datos tales como: Psycinfo; Eric, Ebsco Host, Ulrichs' Periodicals; DOAJ; Latindex; DICE; así como el in- RECS. Ha publicado 9 monografías, así como 7 actas de congresos, todas ellas de carácter internacional, y un total de 28 capítulos de libro). En la actualidad, forma parte del equipo trabajo de un proyecto I+D sobre el uso de la realidad aumentada para la detección e intervención en violencia entre iguales y consumo de sustancias en estudiantes de educación secundaria. En cuanto a ponencias y aportaciones a congresos y jornadas se refiere, han sido 36 las aportaciones. Respecto a otros méritos de investigación, destaca: la pertenencia a comités de organización de diferentes congresos internacionales, miembro de 2 comités de edición/redacción de revistas científicas, la European Journal of Health Research y la European Journal of Child Development, Education and Psychopathology. La participación que ha tenido en cursos y congresos ha sido numerosa.

Orcid ID: https://orcid.org/0000-0002-4297-6824

Google Scholar: https://scholar.google.es/citations?hl=es\&user=Avf9nsQAAAAJ 